\title{
Nonalcoholic steatohepatitis: a toxic liver disease in industrial workers
}

Cotrim HP, Andrade ZA, Parana R, Portugal M, Lyra LG, Freitas LAR. Nonalcoholic steatohepatitis: a toxic liver disease in industrial workers. Liver 1999: 19: 299-304. (C) Munksgaard, 1999

Abstract: Aims: Occupational/environmental exposure to hepatotoxins has recently been implicated in nonalcoholic steatohepatitis (NASH). The aims of this study were to determine the presence and frequency of NASH in a large group of workers chronically exposed to several volatile petrochemical products in an industrial area in north-east Brazil and to observe its course in workers removed from the work environment. Methods: 1500 asymptomatic workers were screened with standard liver blood tests during 1994-5. Those with elevated transaminases ( $>3 \times$ normal) on 3 occasions were evaluated further both clinically and with serum $\mathrm{HBsAg}$, anti-HCV, ferritin, lipids and autoantibody determination. Patients with either no etiological diagnosis, positive $\mathrm{HBsAg} / \mathrm{anti}-\mathrm{HCV}$ serology and/or excess alcohol intake underwent liver biopsy. Those with obesity, diabetes or an isolated abnormal GGT were excluded. Of workers diagnosed as having NASH (compatible histology and no excess alcohol intake), a proportion were removed from the work environment and evaluated monthly with liver blood tests and a repeat liver biopsy 8-14 months later. Results: 112 workers had abrormal transaminases and 32 fulfilled the criteria for liver biopsy. 20 of these were classified as NASH, the remainder had viral hepatitis $(n=6)$, alcoholic liver disease $(n=5)$ or portal vein thrombosis $(n=1)$. In all of the $10 / 20$ who were removed from the work environment, their aminotransferases and GGT gradually decreased and their histology improved. Conclusions: These results demonstrate that NASH can occur following chronic exposure to volatile petrochemical substances in the workplace. Exposed workers should be regularly screened for the presence of liver damage and ideally removed from the work environment where possible.

\author{
Helma P. Cotrim 1 , \\ Zilton A. Andrade ${ }^{2}$, \\ Raimundo Parana', \\ Marcelo Portugal', Luiz G. Lyra' \\ and Luiz A. R. Freitas ${ }^{2}$
}

Division the Gastroenterology, Department of Medicine, Universidade Federal da Bahia ${ }^{1}$ and CPqGM-FIOCRUZ², Bahia, Brazil

Key words: nonalcoholic steatohepatitis hepatotoxicity - toxic liver injury environmental hepatotoxins

Helma P. Cotrim, M.D., Serviço de GastroHepatologia, Hospital Universitário Prof. Edgar Santos, $6^{\circ}$. andar. Rua Augusto Viana, $S / N$, Canela, CEP: $40.000-000$ Salvador, Bahia, Brazil

Received 16 June 1998

Accepted for publication 13 January 1999
The spectrum of liver diseases produced by environmental toxins ranges from sub-clinical hepatic dysfunction to massive hepatic necrosis. The degree of hepatic damage depends on dose, duration of exposure, the activity of hepatic drug metabolizing enzymes and individual susceptibility (1-4). Occupational or environmental exposure to hepatotoxins has recently been included among the increasing number of associations with nonalcoholic steatohepatitis (NASH) $(5,6)$, a condition which has been primarily described in obese and/or diabetic females $(7,8)$. The existence and clinical importance of "occupational" NASH is however not well established. The aims of this study were therefore twofold; first, to determine the presence and frequency of NASH in a large group of petro- chemical workers in north-east Brazil, second, to observe the course of NASH in workers removed from the industrial area and thereby determine the likelihood that any NASH detected was due to petrochemical exposure.

\section{Material and methods}

Study design

The study included two protocols. The first prospectively evaluated asymptomatic workers with elevated levels of aspartate and alanine aminotransferases from a large industrial area of northeast Brazil. They were chronically exposed to several volatile petrochemical products, including benzene, dimethylformamide, ethylene, vinyl chloride and 


\section{Cotrim et al.}

others (see appendix) for at least 5 years. The second protocol was a follow-up study of workers with histologically confirmed NASH, who had been removed from the industrial area.

\section{Study group selection}

The population under study consisted of 1500 petrochemical workers undergoing routine yearly evaluation by standard blood liver tests. Workers were initially selected on the basis of having aspartate and alanine aminotransferases (AST, ALT) and/or gamma-glutamyltransferase (GGT) levels at least 1.5 times the upper limits of normal on three or more determinations within 5 months between May 1994 and December 1995.

\section{Evaluation}

The initial evaluation included a complete clinical history, physical examination and laboratory tests. All subjects answered a questionnaire concerning alcohol intake, drug use, duration of employment and potential exposure to industrial or petrochemical products. Standard liver blood tests, lipids, glucose, HBsAg (AUSRIA, Abbott Labora-

Table 1. Demographic characteristics and liver blood tests in patients with NASH $(n=20)$

\begin{tabular}{lc} 
Age, yr & Mean $=36$ (range $31-56)$ \\
Gender & Maie: $19(95 \%)$ \\
& Female: $1(5 \%)$ \\
Duration of employment $(y r)$ & Mean $=10$ (range $5-25)$ \\
Alcohol consumption & None or Occasional \\
Range of elevated liver enzymes & (x upper limits of normal) \\
AST & 1.5 to 3.0 \\
ALT & 1.5 to 5.0 \\
GGT & 1.5 to 7.0 \\
Ratio ALT/AST $>1$ & $20(100 \%)$ \\
\hline
\end{tabular}

Table 2. Frequency of histological changes and their intensity in 20 active petrochemical workers with the diagnosis of NASH

\begin{tabular}{lcccc}
\hline Lesions & Severe & Moderate & Mild & Absent \\
\hline $\begin{array}{l}\text { Steatosis } \\
\text { Microvesicular }\end{array}$ & 1 & 8 & 9 & 2 \\
$\quad \begin{array}{l}\text { Macrovesicular } \\
\text { Hepatocellular }\end{array}$ & 6 & 6 & 7 & 1 \\
$\quad$ Balooning & 1 & 11 & 8 & 0 \\
Inflammation & & & & \\
$\quad$ Portal & 0 & 4 & 13 & 3 \\
Acinar & 0 & 2 & 18 & 0 \\
Fibrosis & & & & \\
Portal & 0 & 5 & 11 & 4 \\
$\quad$ Perisinusoidal & 1 & 1 & 4 & 14 \\
Mallory bodies & 0 & 0 & 0 & 0 \\
\hline
\end{tabular}

tories, N Chicago, IL), anti-HCV (RIBA III, Chiron, Emeryville, CA), ferritin and autoantibodies (anti-nuclear, anti-mitochondrial, anti-smooth muscle) were routinely performed. Workers with either no etiological diagnosis, positive $\mathrm{HBsAg} /$ anti-HCV or a history of excessive alcohol intake $(>60-80$ g/day or $>3$ times per week) were subjected to liver biopsy. Those with obesity (body mass index $>10 \%$ normal) and/or diabetes or an isolated raised GGT were excluded from histological evaluation.

\section{Follow-up group}

Workers with steatohepatitis on liver biopsy and no history of alcohol excess were diagnosed as suffering from NASH. A proportion of these workers were removed from the industrial area by mutual consent. The remainder refused to be moved for personal/economic reasons. The removed workers

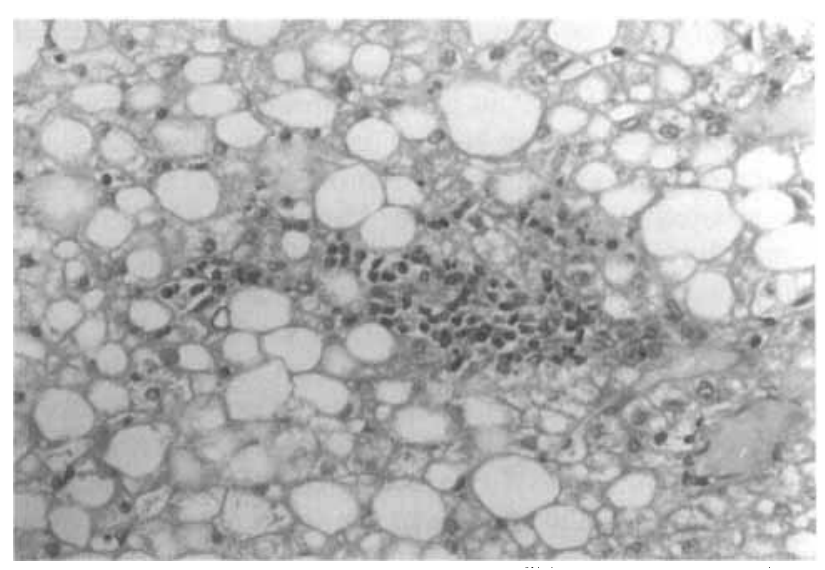

Fig. 1. Macro- and microvesicular-steatosis with inflammatory infiltrate (Hematoxylin \& Eosin $\times 200$ ).

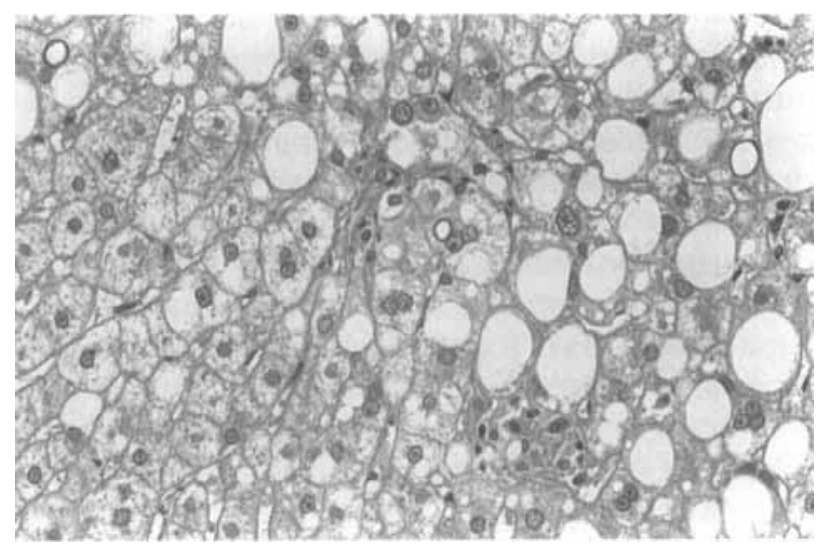

Fig. 2. Although the general structure of the parenchyma is maintaind the hepatocytes exhibit different degrees of changes such as ballooning, macro and micro vacuolation. (Hematoxylin \& Eosin $\times 400$ ). 


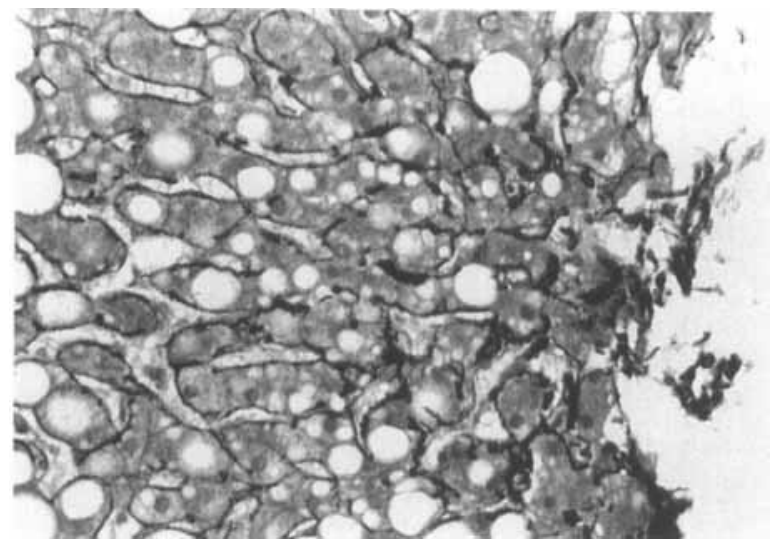

Fig. 3. Perisinusoidal fibrosis appears as dense fibers of collagen thickening of the sinusoidal walls in this focal area of the hepatic parenchyma. (Sirus-red $\times 400$ ).

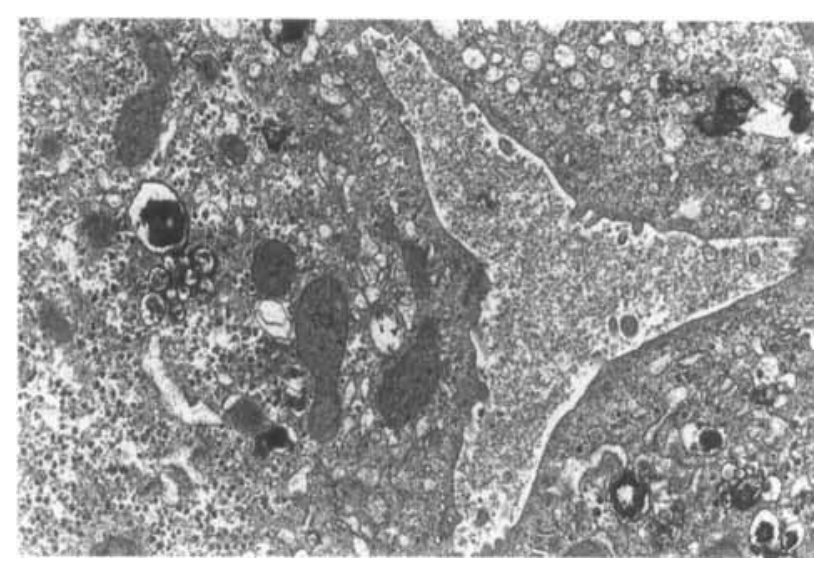

Fig. 4. Dilatation of biliary canaliculi, loss of microvilli, peribiliary granules. (Electron microscopy $\times 7000$ ).

were evaluated monthly with AST, ALT and GGT determinations and with repeat liver biopsy at a mean interval of 1 year (range 8-14 months).

\section{Morphological study}

Hepatic tissue was examined by light and transmission electron microscopy. Paraffin sections of Bouin-fixed materials were stained with hematoxylin-eosin, sirius-red for collagen, periodic acid Schiff (PAS), with and without previous diastase digestion, Perls' method for iron and Gomori's reticulum. For electron microscopy, tiny fragments were immediately fixed in cold $2 \%$ glutaldehyde in $0.1 \mathrm{M}$ cacodylate buffer for $1-2 \mathrm{~h}$, post fixed in $1 \%$ osmium tetroxide, washed in the buffer, dehydrated in graded acetone and embedded in Epon resin (Polybed-812TM). Ultra thin sections from selected areas were cut with a diamond knife, mounted on copper grids and contrasted with uranyl acetate-lead citrate. Observations were carried out on a Zeiss EM-109 electron microscope at 50 Kv. Biopsies were examined by two independent pathologists blinded as to whether they were index or follow-up cases.

\section{Results}

112 workers fulfilled the criteria for initial inclusion in the study and 32 the criteria for liver biopsy evaluation. Twenty of the 32 biopsied subjects were classified as having NASH on the basis of compatible histology and the absence of a significant alcohol history. Their major characteristics are shown in Table 1.

The other 12 individuals biopsied included 6 with features of chronic viral hepatitis, (5 anti$\mathrm{HCV}$ positive, $1 \mathrm{HBsAg}$ positive), one with evidence of intra-hepatic portal vein thrombosis and 5 who had a history of excessive alcohol intake and had diagnosis of alcoholic liver disease.

Histological findings in the 20 NASH patients showed that the liver maintained its normal architecture, but almost all hepatocytes appeared clear, distended and many of them showed macro and micro vacuolation, with no particular acinar distribution (Fig. 1). Single-cell necrosis with focal leukocyte accumulation was rarely seen. The hepatocytes exhibited different degrees of ballooning (Fig. 2). Mallory bodies were not detected. Infiltration with inflammatory cells occurred in portal spaces and in small foci within the parenchyma. Inflammation was always mild, rarely of a moderate degree. A few polymorphonuclears were seen but most of the inflammatory cells were macrophages and lymphocytes. Perisinusoidal and portal fibrosis was also observed (Fig. 3). Perisinusoidal collagens fibers were increased in amount and thickness, and sinusoidal cells appeared hyperplastic. A quantitative evaluation of all these features appears in Table 2.

Ultrastructurally, mild to severe signs of hepatic injury were observed in all cases studied. In addition to macro and microvesicular steatosis of hepatocytes, there were frequent dilatation, blebs, bile plugging and paucity of microvilli in bile capillaries. Frequent electron dense bodies of variable sizes and shapes (lipofuscin), some presenting concentric laminated dark material (residual bodies), were also seen. Lipolysosomes were seen around dilated bile canaliculi (Fig. 4). Marked dilatation of both smooth and rough endoplasmic reticulum, enlarged and bizarre-shaped mitochondria, and megamitochondria, sometimes with paracrystalline inclusions, were frequently seen (Fig. 5). Hepatic stellate cells were enlarged and more numerous than usual.

Ten out of the 20 workers with NASH were re- 


\section{Cotrim et al.}

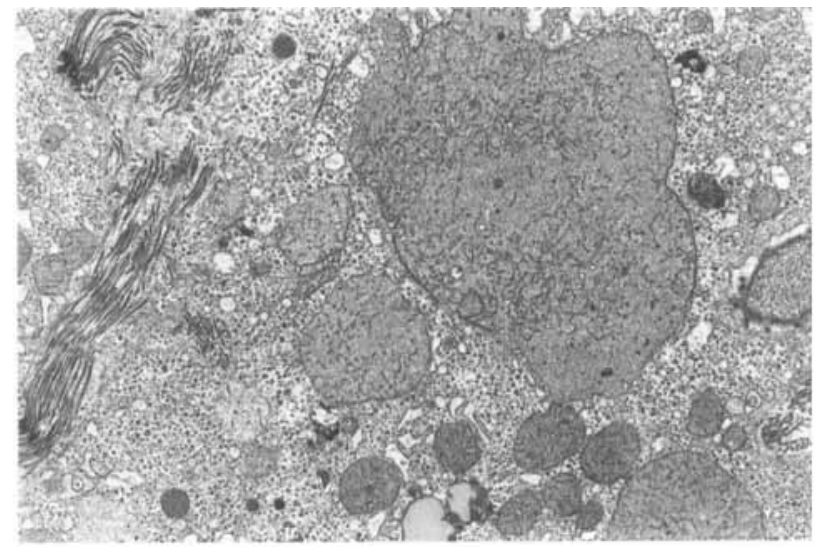

Fig. 5. Mitochondria enlarged with bizarre forms and abnormal sizes, megamitochondria, paracrystalline inclusions. (Electron microscopy $\times 7000$ ).

moved from the petrochemical industrial area. All of them continued to be asymptomatic throughout the follow-up period. All but one were male, ages varying from 31 to 56 years (mean 36 ). The duration of petrochemical exposure ranged from 9 to 20 years (mean 13). Serum levels of albumin, cholesterol, glucose and prothrombin time remained normal. Levels of AST, ALT and GGT gradually decreased in all the workers. The serum AST levels varied from

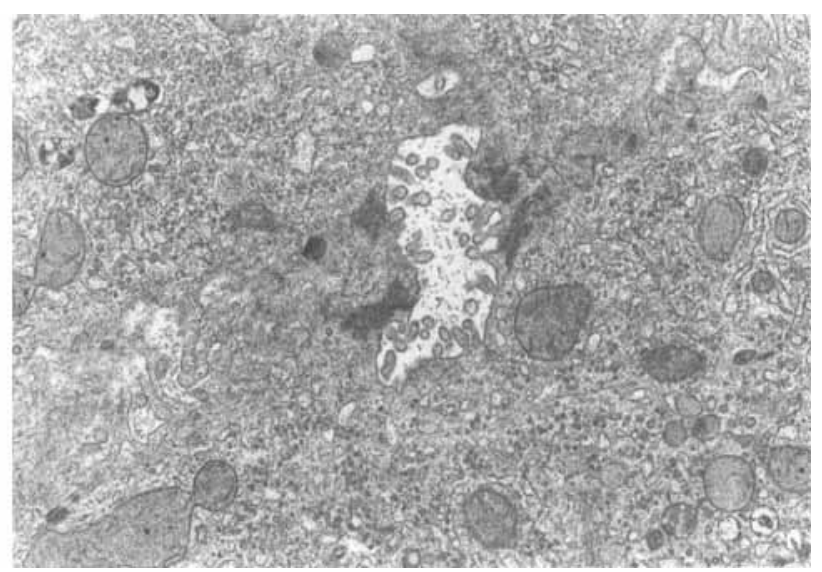

Fig. 6. Electron microscopy from the liver of a patient removed from industrial area. Normal bile canaliculus. Microvilli are well preserved. (Electron microscopy $\times 4000$ ).

normal to 1.5 times normal and ALT and GGT from normal to 2.5 times normal. Repeat liver biopsy showed improvement of liver structure in all cases, although with variable degrees (Table 3 ). Regression of macro and microvesicular steatosis and liver-cell ballooning degeneration were the most significant changes. In addition, portal inflammation decreased or disappeared as did fibrosis. The improvement of hepatic structure was also evident at the ultrastructural level (Fig. 6).

Table 3. Grading of histological findings in liver biopsies made in subjects with NASH, before and after removal from a petrochemical plant

\begin{tabular}{|c|c|c|c|c|c|c|c|c|}
\hline \multirow[b]{2}{*}{ No. } & \multicolumn{2}{|c|}{ Steatosis } & \multirow[b]{2}{*}{ Ballooning } & \multicolumn{2}{|c|}{ Inflammation } & \multicolumn{2}{|c|}{ Fibrosis } & \multirow[b]{2}{*}{ Total } \\
\hline & Macro & Micro & & Acinar & Portal & Portal & Perisinusoidal & \\
\hline $1 a$ & 1 & 1 & 0 & 1 & 2 & 1 & 1 & 7 \\
\hline $1 b$ & 1 & 0 & 0 & 0 & 0 & 0 & 0 & 1 \\
\hline $2 a$ & 2 & 2 & 2 & 1 & 2 & 1 & 1 & 11 \\
\hline $2 b$ & 1 & 1 & 1 & 1 & 2 & 1 & 1 & 8 \\
\hline $3 a$ & 3 & 2 & 2 & 1 & 2 & 2 & 2 & 13 \\
\hline $3 b$ & 2 & 2 & 2 & 1 & 2 & 1 & 0 & 10 \\
\hline $4 a$ & 2 & 2 & 2 & 1 & 1 & 0 & 0 & 8 \\
\hline 40 & 0 & 0 & 0 & 0 & 0 & 0 & 0 & 0 \\
\hline $5 a$ & 3 & 2 & 2 & 1 & 1 & 0 & 1 & 10 \\
\hline $5 b$ & 2 & 1 & 1 & 1 & 1 & 0 & 1 & 7 \\
\hline $6 a$ & 3 & 1 & 2 & 1 & 2 & 1 & 1 & 11 \\
\hline $6 b$ & 3 & 1 & 1 & 0 & 1 & 0 & 1 & 7 \\
\hline $7 \mathrm{a}$ & 1 & 1 & 1 & 1 & 1 & 0 & 0 & 5 \\
\hline $7 \mathrm{~b}$ & 1 & 0 & 0 & 0 & 0 & 0 & 0 & 1 \\
\hline $8 a$ & 1 & 1 & 2 & 1 & 1 & 0 & 1 & 7 \\
\hline $8 b$ & 1 & 0 & 1 & 1 & 0 & 0 & 1 & 4 \\
\hline $9 a$ & 3 & 3 & 2 & 1 & 1 & 0 & 1 & 11 \\
\hline $9 b$ & 1 & 1 & 0 & 1 & 1 & 0 & 1 & 5 \\
\hline $10 \mathrm{a}$ & 2 & 1 & 2 & 1 & 1 & 0 & 0 & 7 \\
\hline $10 \mathrm{~b}$ & 1 & 0 & 0 & 1 & 0 & 0 & 0 & 2 \\
\hline
\end{tabular}

$a=$ first biopsy. $b=$ after removal from working area. $1=$ mild. $2=$ moderate. $3=$ severe. 


\section{Discussion}

Nonalcoholic steatohepatitis is a chronic liver disease found frequently in obese women with diabetes. It has been defined by liver biopsy features and has the appearance of alcoholic hepatitis. (710 ). More recently, NASH has been considered in other patients (6), and occupational exposure to hepatotoxins has been included among its etiologic factors (5). Establishing a definite causal relationship between any form of hepatotoxicity and environmental/occupational exposure is difficult, and evidence is often purely circumstantial (11-14). We believe that this study provides strong evidence that chronic exposure to petrochemicals can lead to liver injury of the "NASH" type. All workers had a history of prolonged periods of exposure to potentially toxic substances that are recognized to cause liver injury $(1,2,4)$. Excessive alcohol intake and other causes of steatohepatitis such as obesity and diabetes were carefully excluded and the ALT/ AST ratio $>1$ in all the patients also supports a non-alcoholic etiology (15). In addition to steatohepatitis, all included subjects demonstrated histological features compatible with toxic injury including the presence of giant and bizarre mitochondria, proliferation and dilatation of smooth endoplasmic reticulum, lipofuscinosis, an increase in the number of stellate cells and perisinusoidal fibrosis (16). Finally, perhaps the best evidence incriminating occupational exposure to petrochemicals as the cause of the liver injury was the improvement in biochemistry and histology in all workers removed from the industrial area.

Clearly this study does not allow us to draw any conclusions on the natural history and prognosis of NASH related to petrochemical exposure. However recent studies have suggested that NASH due to other etiologies may progress to fibrosis and cirrhosis (6). It would therefore seem pertinent to periodically monitor liver blood tests in workers chronically exposed to petrochemicals and advise removal from the work environment in workers with evidence of liver damage. Recent evidence supporting a role for oxidative stress and lipid peroxidation in the pathogenesis of NASH (17) suggests that antioxidants may be a therapeutic option in those affected workers unable to avoid further exposure.

\section{Acknowledgements}

The authors thank Christopher P. Day, M.D., Ph.D and Professor Oliver F. W. James from University of Newcastle Upon Tyne, England for their assistance with preparation of the manuscript.

Abbreviations: NASH, nonalcoholic steatohepatitis; ALT, alanine aminotransferases; AST, aspartate aminotransferases;
GGT, gamma-glutamyl transferases; HBsAg, hepatitis B surface antigen; anti-HCV, antibody to hepatitis $\mathrm{C}$ virus; $\mathrm{H}$, hematoxylin; E, eosin; EM, electron microscopy.

\section{Appendix}

Petrochemical products most frequently found at the industrial area in this study.

- Acrylonitrile

- Acetylene

- Benzene

- Carbon tetrachloride

- Chloroform

- Chloroethyl vinyl ether

- Dimethylformamide

- Ethane

- Ethylene

- Hexane

- Methanol

- Toluene

- Trinitrotoluene

- Tetrachloroethane

- Vinyl Chloride

- Styrene

- Xylenes

- Hydrocarbon mists.

\section{References}

1. Tamburro C H. Chemical hepatitis. Pathogenesis, detection and management. Med Clin North Am 1979; 63: 54566.

2. Popper H, Gebber M A, Shaffner F, Selikoff I J. Environmental hepatic injury in man. In: Popper $\mathrm{H}$, Schaffner F, eds. Progress in liver diseases. New York: Grune \& Stratton, 1979; 6: 605-38.

3. Zimmerman J H. Hepatotoxicity. Dis Mon 1993; 39: 673788.

4. Farrell G C. Liver disease due to environmental toxins in liver disease. In: Farrell GC, ed. Drug-induced liver disease. Edinburgh London: Churchill Livingstone, 1994; 511-5.

5. Neuschwander-Tetrı B A, Bacon B B. Nonalcoholic Steatohepatitis. Med Clin North Am 1996; 80: 1147-66.

6. BaCON B R, Farahvash M J, Janney C G, NeuschwandER-TETRI B A. Nonalcoholic steatohepatitis: an expanded clinical entity. Gastroenterology 1994; 107: 1103-9.

7. LEE R G. Nonalcoholic steatohepatitis. A study of 49 patients. Hum Pathol 1989; 20: 594-8.

8. Powell E E, Cooksley W G E, Hanson R, Searle J, HalLIDAY J W, Powell L W. The natural history of nonalcoholic steatohepatitis: a follow-up study of forty-two patients for up to 21 years. Hepatology $1990 ; 11: 74-80$.

9. Diehl A N, Goodman Z, Ishak K G. Alcohollike liver disease in nonalcoholics. Gastroenterology 1988; 95: 1056-62.

10. Ludwig J, Yiggiano T R, McGill D B, OTt B J. Nonalcoholic steatohepatitis. Mayo clinic experiences with a hitherto unnamed disease. Mayo Clin Proc 1980; 55: 434-8.

11. Dossing M, RANEK L. Isolated liver damage in chemical workers. Br J Ind Med 1984; 41: 142-4.

12. Mitchell J W. Screening for hepatic damage. J Occup Med $1987 ; 31: 266-7$.

13. Wright C, Rivera J C, Baetz J H. Liver function testing in a working population: three strategies to reduce false positive results. J Occup Med 1988; 30: 693-7. 


\section{Cotrim et al.}

14. Tamburro $\mathrm{C} \mathrm{H}$, Liss G M. Tests for hepatotoxicity: usefulness in screening workers. J Occup Med 1986; 28: $1034-44$.

15. Itoh S, Youngel T, Kawagoe K. Comparison between nonalcoholic steatohepatitis and alcoholic hepatitis. Am J Gastroenterol 1987; 8: 650-7.

16. Phillips M J, Poucell S, Patterson J, Valencia P. Drugs and toxic effects. In: Phillips M J, Poucell S, Patterson J, Valencia P, eds. The Liver. An atlas and text of ultrastructural pathology. New York: Raven Press, 1987: 159 71.

17. DAY C P, JAMes O F W. Steatohepatitis - a tale of two "hits"? Gastroenterology 1998; 114: 842-5. 